\title{
High-Convexity Tightness Predicts the Shunt Response in Idiopathic Normal Pressure Hydrocephalus
}

\author{
(D) W. Narita, (D). Nishio, (D). Baba, (D). lizuka, (D). Ishihara, (D). Matsuda, (D) M. Iwasaki, (D). Tominaga, and (DE. Mori
}

\begin{abstract}
BACKGROUND AND PURPOSE: Although neuroimaging plays an important role in the diagnosis of idiopathic normal pressure hydrocephalus, its predictive value for response to shunt surgery has not been established. The purpose of the current study was to identify neuroimaging markers that predict the shunt response of idiopathic normal pressure hydrocephalus.
\end{abstract}

MATERIALS AND METHODS: Sixty patients with idiopathic normal pressure hydrocephalus underwent presurgical brain MR imaging and clinical evaluation before and 1 year after shunt surgery. The assessed MR imaging features included the Evans index, high-convexity tightness, Sylvian fissure dilation, callosal angle, focal enlargement of the cortical sulci, bumps in the lateral ventricular roof, and deep white matter and periventricular hyperintensities. The idiopathic normal pressure hydrocephalus grading scale total score was used as a primary clinical outcome measure. We used measures for individual symptoms (ie, the idiopathic normal pressure hydrocephalus grading scale subdomain scores, such as gait, cognitive, and urinary scores), the Timed Up and Go test, and the Mini-Mental State Examination as secondary clinical outcome measures. The relationships between presurgical neuroimaging features and postoperative clinical changes were investigated by using simple linear regression analysis. To identify the set of presurgical MR imaging features that best predict surgical outcomes, we performed multiple linear regression analysis by using a bidirectional stepwise method.

RESULTS: Simple linear regression analyses demonstrated that presurgical high-convexity tightness, callosal angle, and Sylvian fissure dilation were significantly associated with the 1-year changes in the clinical symptoms. A multiple linear regression analysis demonstrated that presurgical high-convexity tightness alone predicted the improvement of the clinical symptoms 1 year after surgery.

CONCLUSIONS: High-convexity tightness is a neuroimaging feature predictive of shunt response in idiopathic normal pressure hydrocephalus.

ABBREVIATIONS: DESH = disproportionately enlarged subarachnoid space hydrocephalus; $\mathrm{DWMH}=$ deep white matter hyperintensity; iNPH $=$ idiopathic normal pressure hydrocephalus; iNPHGS = idiopathic normal pressure hydrocephalus grading scale; MMSE = Mini-Mental State Examination; PVH = periventricular hyperintensity; TUG = Timed Up and Go test

diopathic normal pressure hydrocephalus (iNPH) has been increasingly recognized as a common cause of gait disturbance and cognitive impairment in elderly individuals. The prevalence of iNPH is estimated to be $1.1 \%-2.1 \%,^{1,2}$ which is greater than

Received February 10, 2016; accepted after revision April 18.

From the Departments of Behavioral Neurology and Cognitive Neuroscience (W.N., Y.N., T.B., O.I., T.I., M.M., E.M.) and Neurosurgery (M.I., T.T.), Tohoku University School of Medicine, Sendai, Japan.

Drs Narita and Nishio had full access to all the data in the study and take responsibility for the integrity of the data and the accuracy of the data analysis. Contributions were the following: study concept and design, Narita, Nishio; acquisition, analysis, or interpretation of data, all authors; drafting of the manuscript, Narita, Nishio; critical revision of the manuscript for important intellectual content, all authors; statistical analysis, Narita, Nishio; obtained funding, Mori; administrative, technical, or material support: Baba, Ishihara, lizuka, Matsuda, Iwasaki, Tominaga, Mori; study supervision. Tominaga, Mori.

This study was supported by a Health and Labor Sciences Research Grant for Research on Intractable Diseases, Ministry of Health, Labor and Welfare. that of Parkinson disease (approximately $1 \%$ in those older than 60 years of age). ${ }^{3}$ Differentiating iNPH from neurodegenerative diseases is critical because effective surgical treatment is available for iNPH. Neuroimaging plays an important role in the differential diagnosis of iNPH. Specifically, disproportionately enlarged subarachnoid space hydrocephalus (DESH) on MR imaging or CT is now accepted as a useful diagnostic marker. ${ }^{4,5}$

Shunt surgery is the criterion standard treatment for iNPH.

Please address correspondence to Wataru Narita, MD, Department of Behavioral Neurology and Cognitive Neuroscience, Tohoku University School of Medicine, 2-1, Seiryo-machi, Aoba-ku, Sendai 980-8575, Japan; e-mail: naritaw@med.tohoku.ac.jp, or Yoshiyuki Nishio, MD, PhD, Department of Behavioral Neurology and Cognitive Neuroscience, Tohoku University School of Medicine, 2-1, Seiryomachi, Aoba-ku, Sendai 980-8575 Sendai, Japan; e-mail: nishiou@med.tohoku.ac.jp -- Indicates open access to non-subscribers at www.ajnr.org

三 Indicates article with supplemental on-line table.

http://dx.doi.org/10.3174/ajnr.A4838 
Although symptomatic improvement following shunt surgery is observed in up to $70 \%$ of patients, the effectiveness varies from patient to patient. ${ }^{6,7}$ Although predictive markers for surgical outcomes are necessary for decision-making regarding the surgical indications, this need is currently unmet. For example, the sensitivity and specificity of the CSF tap tests, which have been widely used to predict shunt response in clinical practice, ${ }^{1,8}$ remain at 42\%-93\% and 20\%-100\%, respectively. ${ }^{9-12}$ A previous study demonstrated that elevated overnight intracranial pressure pulse amplitude and CSF pulse amplitude during lumbar infusion predicted better shunt response. ${ }^{13}$ However, these procedures are invasive and not widely available. To explore an easily available marker predictive of shunt response, the current study investigated presurgical neuroimaging features associated with better surgical outcome in iNPH.

\section{MATERIALS AND METHODS}

This study was conducted in accordance with the Declaration of Helsinki, and the protocol was approved by the ethics committee of the Tohoku University Hospital. Written informed consent was obtained from all participants.

\section{Diagnosis of iNPH}

Although several diagnostic criteria for iNPH have been proposed, there is still lack of consensus on how to diagnose iNPH preoperatively. ${ }^{8,14}$ It is indispensable to determine the presence or absence of comorbid neurologic diseases, such as Alzheimer disease, Parkinson disease, and cerebrovascular diseases and their contributions to clinical symptoms, to select appropriate candidates for shunt surgery. The presence of DESH alone is not informative enough to know whether hydrocephalus is the primary pathology associated with clinical symptoms because $>60 \%$ of those who have DESH on MR imaging do not have any of the triad of symptoms. ${ }^{15}$ Thus, we made the diagnosis of iNPH on the basis of comprehensive symptomatic and neuroimaging investigations. Our diagnostic procedures were as follows:

1) All patients who were referred to the Department of Behavioral Neurology and Cognitive Neuroscience of Tohoku University Hospital due to progressive cognitive impairment and/or gait disturbance underwent neurologic and neuropsychological examinations, routine laboratory testing, brain MR imaging, and/or CT. When patients exhibited $\geq 1$ of the triad of symptoms and neuroimaging features of DESH, they were diagnosed as having probable iNPH ${ }^{1,14}$ and were invited for inpatient evaluation.

2) The patients who were admitted to the Department of Behavioral Neurology and Cognitive Neuroscience were given comprehensive neurologic and neuropsychological assessments by behavioral neurologists and speech-language pathologists, and they underwent $3 \mathrm{D}$ volumetric MR imaging or CT, single-photon emission CT, and a CSF tap test.

3) If patients exhibited clinical and/or neuroimaging features pathognomonic of neurologic disease other than iNPH (eg, severe and dissociated amnesia suggestive of Alzheimer disease and severe sympathetic denervation on iodine 123 metaiodobenzylguanidine myocardial scintigraphy indicative of Parkinson disease or dementia with Lewy bodies), they were excluded as candidates for surgical intervention.
Table 1: Presurgical clinical characteristics of patients $(n=60)^{a}$

\begin{tabular}{lc}
\hline \multicolumn{1}{c}{ Characteristics } & \\
\hline Age (yr) & $76.4(3.8)$ \\
Sex, male & $34(57 \%)$ \\
Education (yr) & $10.2(3.0)$ \\
Duration of symptoms (yr) & $3.3(1.6)$ \\
LP shunt & $23(38 \%)$ \\
Medical history & \\
Hypertension & $36(60 \%)$ \\
Diabetes & $18(30 \%)$ \\
Lipid disorder & $18(30 \%)$ \\
Current smoker & $6(10 \%)$ \\
Prevalence of symptoms & \\
Triad (all 3 symptoms) & $27(45 \%)$ \\
Gait and cognitive & $21(35 \%)$ \\
Gait and urinary & $3(5 \%)$ \\
Cognitive and urinary & $1(2 \%)$ \\
Gait only & $5(8 \%)$ \\
Cognitive only & $2(3 \%)$ \\
Urinary only & 0 \\
\hline
\end{tabular}

Note:-LP indicates lumboperitoneal.

${ }^{a}$ Age, education, and the duration of symptoms are indicated as the means (SDs). The other variables are indicated as the number of patients (\%).

${ }^{b}$ Each symptom was regarded as positive when the corresponding score of the iNPH grading scale was $\geq 2$.

4) Given the insufficient sensitivity of the CSF tap test, we recommended shunt surgery to patients with clinical and neuroimaging features suggestive of $\mathrm{iNPH}$ without comorbid neurologic diseases regardless of the response to the tap test.

5) Patients with previous histories of subarachnoid hemorrhage, meningitis, or head injury and those with neuroimaging evidence of aqueductal stenosis or a Blake pouch cyst were diagnosed as having secondary normal pressure hydrocephalus and were not included in the study.

\section{Selection of Surgical Methods}

In the earlier part of this study, ventriculoperitoneal shunt was preferentially performed in our institution. In the last half of the study, we performed lumboperitoneal shunt unless contraindications were present or patients requested ventriculoperitoneal shunt. The contraindications for lumboperitoneal shunt included severe spinal canal stenosis or lumbar spine deformity, which was diagnosed on the basis of neurologic examination, spinal MR imaging, and observations on lumbar puncture.

\section{Subjects}

We identified 103 consecutive patients with iNPH who were admitted to the Department of Behavioral Neurology and Cognitive Neuroscience and underwent shunt surgery between December 19, 2005, and May 13, 2013. Of these 103 consecutive patients, we retrospectively selected 60 patients who underwent presurgical MR imaging evaluation and completed 1-year postsurgical follow-up. The demographic and clinical profiles of the patients are summarized in Table 1. Forty-three patients were excluded from the study for the following reasons: Two patients died, 4 developed shunt system problems, 2 developed pneumonia, 1 developed a femoral fracture, 1 developed a cerebral infarction, 16 moved to hospitals that were nearer to their homes, 13 did not return for follow-up visits for other reasons, and 4 were excluded 


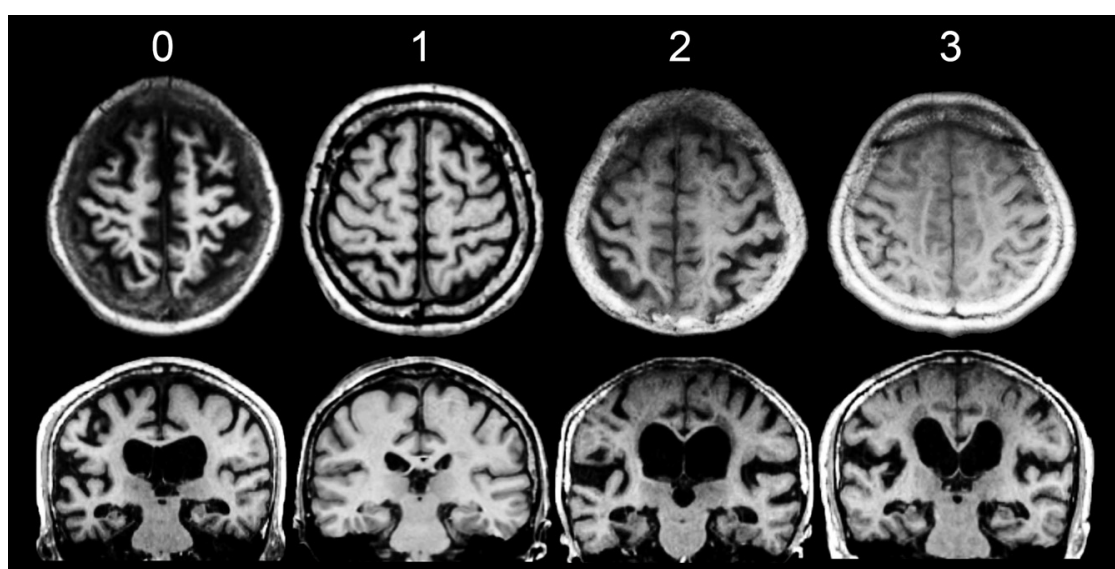

A

$\begin{array}{llll}0 & 1 & 2 & 3\end{array}$

B

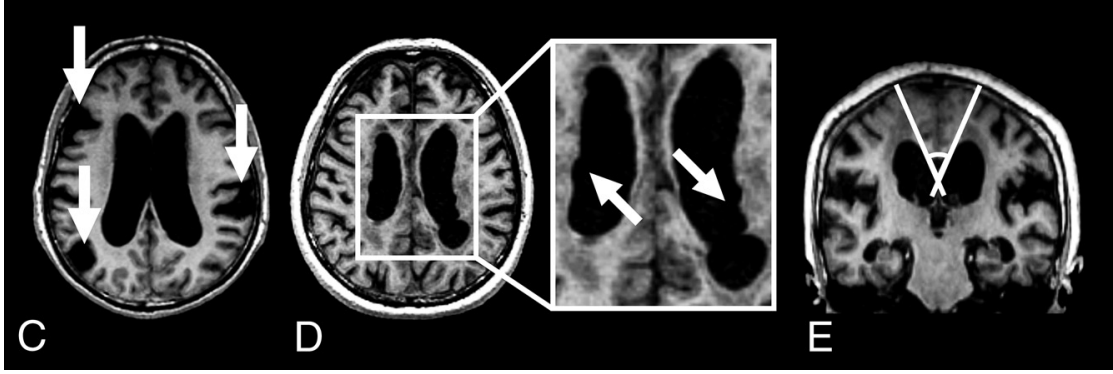

FIG 1. Visual rating scales for neuroimaging features in iNPH. A, High-convexity tightness: 0 , dilated; 1 , normal; 2 , mildly tight; 3 , severely tight. $B$, Sylvian fissure dilation: 0 , narrowed; 1 , normal; 2 , mildly dilated; 3 , severely dilated. $C$. Focal dilation of the sulci (indicated by the arrows). D, Bumps in the lateral ventricular roof (indicated by the arrows). E, Callosal angle.

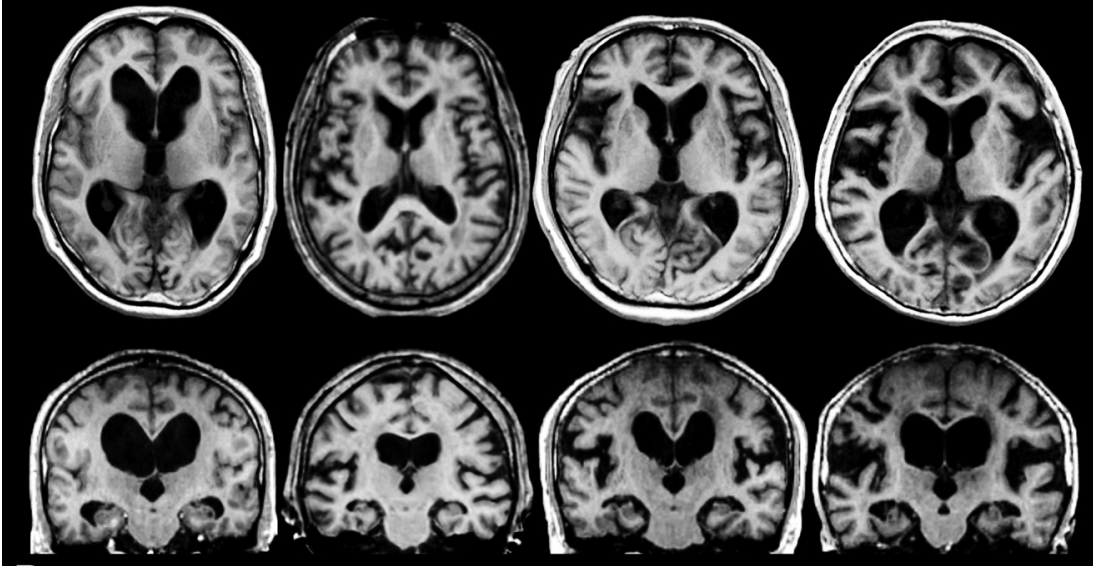

sures for individual symptoms of the classic triad as secondary outcome measures:

1) Gait was assessed with the iNPHGS gait score and the Timed Up and Go test (TUG). ${ }^{17}$

2) Cognitive function was assessed with the iNPHGS cognitive score and the Mini-Mental State Examination (MMSE). ${ }^{18}$

3) Urinary function was assessed with the iNPHGS urinary score.

\section{Neuroimaging}

Neuroimaging analyses were conducted on axial and coronal reconstructed images of $3 \mathrm{D}$ volumetric MR imaging. In 44 patients, transverse fluid-attenuated inversion recovery images were used for the evaluation of ischemic changes of the brain.

\section{MR Imaging Acquisition and Preprocessing}

3D T1-weighted (transverse 3D-spoiled gradient-recalled: TR, $20 \mathrm{~ms}$; TE, $4.1 \mathrm{~ms}$; thickness, $1.5 \mathrm{~mm}$; FOV, $25 \times 25 \mathrm{~cm}$; and matrix, $256 \times 256)$ and FLAIR (TR, 11,002 ms; TE, $120 \mathrm{~ms}$; thickness, 6.0 $\mathrm{mm}$; gap, $1.0 \mathrm{~mm}$; FOV, $21 \times 21 \mathrm{~cm}$; and matrix, $256 \times 256$ ) images were obtained with a Signa $1.5 \mathrm{~T}$ MR imaging unit (GE Healthcare, Milwaukee, Wisconsin) before shunt surgery. The $3 \mathrm{D}$ T1-weighted images were resliced into sections that were parallel (transverse) and perpendicular (coronal) to the anteroposterior commissural plane and were spaced at 6-mm intervals in each direction.

\section{MR Imaging Measures}

To explore the presurgical MR imaging features that predicted the response to shunt surgery, we used the following measures (Fig 1):

1) The Evans index was calculated as due to incomplete clinical or imaging data. The demographic and clinical characteristics of the excluded patients are shown in the On-line Table.

All the clinical and neuroimaging data described below were gathered in a prospective manner.

\section{Clinical Assessments}

All subjects in this study were evaluated before and 1 year after shunt surgery. The total score of the idiopathic normal pressure hydrocephalus grading scale (iNPHGS), ${ }^{16}$ which represents the global severity of clinical symptoms, was used as a primary outcome measure. We included the following mea- the ratio of the maximum diameter of the frontal horns of the lateral ventricles to the maximum inner diameter of the skull on transverse sections. ${ }^{19}$

2) The callosal angle, the angle between the left and right corpus callosum, was measured on a coronal plane at the posterior commissure. $^{20}$

3) We evaluated the tightness of the high-convexity subarachnoid space on the 4 uppermost contiguous transverse sections and the 3 contiguous coronal sections on and anterior to the posterior commissure. The severity of the high-convexity tightness was visually rated as follows: 0 , dilated; 1 , normal; 2 , mildly tight (tightness was observed over less than three-quarters of the high- 
Table 2: Changes in the clinical symptoms at 1 year ${ }^{\mathrm{a}}$

\begin{tabular}{|c|c|c|c|c|c|c|c|c|c|}
\hline & \multicolumn{2}{|c|}{ Presurgical Score } & \multicolumn{2}{|c|}{ Postsurgical Score } & \multicolumn{2}{|c|}{ Difference } & \multicolumn{3}{|c|}{ Postsurgical Changes (No.) (\%) } \\
\hline & No. & Median (IQR) & No. & Median (IQR) & Median (IQR) & $P$ Value ${ }^{b}$ & Improved & Stable & Deteriorated \\
\hline iNPHGS total [12] & 60 & $6.0(5.0-8.0)$ & 60 & $5.0(3.0-6.0)$ & $1.0(0.5-3.0)$ & $<.001$ & $45(75)$ & $10(17)$ & $5(8)$ \\
\hline iNPHGS gait [4] & 60 & $2.0(2.0-3.0)$ & 60 & $2.0(1.0-3.0)$ & $1.0(0.5-1.0)$ & $<.001$ & $32(53)$ & $24(40)$ & $4(7)$ \\
\hline iNPHGS cognitive [4] & 60 & $2.0(2.0-3.0)$ & 60 & $2.0(1.3-3.0)$ & $0(0-1.0)$ & .001 & $20(33)$ & $36(60)$ & 4 (7) \\
\hline iNPHGS urinary [4] & 60 & $2.5(1.0-3.0)$ & 60 & $1.0(0-1.0)$ & $0(0-1.0)$ & $<.001$ & $29(48)$ & $24(40)$ & $7(12)$ \\
\hline TUG & 55 & $15.1(11.0-20.6)$ & 53 & $11.5(9.2-14.5)$ & $2.5(0.8-4.2)$ & $<.001$ & $35(66)$ & $13(25)$ & $5(9)$ \\
\hline MMSE [30] & 60 & $22.0(20.0-24.8)$ & 59 & $23.0(21.0-27.0)$ & $1(-1-3.0)$ & .014 & $19(32)$ & $32(54)$ & $8(14)$ \\
\hline
\end{tabular}

Note:-IQR indicates interquartile range.

${ }^{a}$ Clinical improvement and deterioration were defined as $\geq 1$-point improvement or deterioration on the iNPHGS, $\geq 10 \%$ reduction or increase in TUG time, and $\geq 3$ points gained or lost on the MMSE. The numbers in square brackets refer to the maximum scores for the tests.

${ }^{b}$ Wilcoxon signed rank test for pre- and postsurgery comparisons.

convexity space); and 3, severely tight (tightness was observed over three-quarters or more of the high-convexity space).

4) The width of the Sylvian fissure was assessed on transverse sections. We used the following visual rating scale: 0 , narrowed; 1 , normal; 2, mildly dilated; and 3, severely dilated on the axial images.

5) The presence (rated as 1) and absence (rated as 0) of focal enlargement of the cortical sulci were visually evaluated on transverse sections.

6) Bumps in the lateral ventricular roof, which are often observed in patients with $\mathrm{iNPH},{ }^{21}$ were visually assessed on transverse sections above the top of the thalamus and rated as present (1) or absent (0).

7) Deep white matter hyperintensities (DWMHs) and periventricular hyperintensities (PVHs) were assessed according to Fazekas et al. ${ }^{22}$

Visual ratings 3-7 were independently evaluated by 2 raters (W.N. and Y.N.) who were blinded to the clinical profiles and surgical outcomes of the patients. The interrater reliability was calculated by using linearly weighted $\kappa$ coefficients. The mean scores of the 2 raters were used for the subsequent analyses.

\section{Statistical Analysis}

The Wilcoxon signed rank test was used to analyze 1-year changes in clinical scores. Simple linear regression analysis was performed to investigate relationships between presurgical neuroimaging features and 1-year changes in the clinical scores (ie, iNPHGS, TUG, and MMSE). Given the exploratory nature of the analysis, no multiple comparison corrections were used.

To identify the set of presurgical MR imaging features that best predicts surgical outcomes, we performed multiple linear regression analysis by using a bidirectional (forward/backward) stepwise method. The outcome variables were 1-year changes of iNPHGS total score (primary outcome measure) and subdomain (gait, cognition, and urinary function) scores (secondary outcome measures). The explanatory variables were the Evans index, high-convexity tightness, Sylvian fissure dilation, and callosal angle on MR imaging. Sex and age were included as nuisance variables. The cutoff $P$ value for inclusion was set at $<.05$ and for exclusion, $<.10$. Statistical analysis was performed by using SPSS version 22.0 (IBM, Armonk, New York).

\section{RESULTS}

\section{Changes in Clinical Symptoms 1 Year after Shunt Surgery}

The results of the clinical assessments before and 1 year after shunt surgery are shown in Table 2. Overall, all clinical measures were significantly improved (Wilcoxon signed rank test, $P<.05$ ). Sev-
Table 3: Presurgical neuroimaging features

\begin{tabular}{lcc}
\hline & $\begin{array}{c}\text { Score }^{\mathrm{a}} \\
\text { (Median [IQR]) }\end{array}$ & $\begin{array}{c}\text { Reliability } \\
\left(\boldsymbol{\kappa}_{\mathrm{w}}\right)\end{array}$ \\
\hline $\begin{array}{l}\text { High-convexity tightness } \\
\text { Sylvian fissure dilation }\end{array}$ & $2.5(2.0-3.0)$ & 0.68 \\
$\begin{array}{l}\text { Focal enlargement of } \\
\text { cortical sulci }\end{array}$ & $3.0(2.5-3.0)$ & 0.50 \\
$\begin{array}{l}\text { Bumps in the lateral } \\
\text { ventricular roof }\end{array}$ & $0.5(0-1.0)$ & 0.27 \\
$\begin{array}{l}\text { DWMHs } \\
\text { PVHs }\end{array}$ & $1.0(0.1-1.0)$ & 0.66 \\
Evans index & $2.0(2.0-3.0)$ & \\
Callosal angle & $2.5(2.0-3.0)$ & 0.71 \\
\hline
\end{tabular}

Note:- $\mathrm{IQR}$ indicates interquartile range; $\kappa_{\mathrm{w}}$, linear weighted $\kappa$ coefficient

a The visual rating scores indicate the mean scores of the 2 raters.

b The DWMHs and PVHs were obtained from 44 patients.

enty-five percent of the patients were improved on the iNPHGS total score; $53 \%$, on the gait score; $33 \%$, on the cognitive score; and $48 \%$, on the urinary score. Improvement of $\geq 3$ points on the MMSE was noted in $32 \%$ of patients.

\section{Presurgical Neuroimaging Characteristics}

The presurgical MR imaging findings are summarized in Table 3. The linearly weighted $\kappa$ coefficients for the visual rating scale were $0.27-0.71$. All of the ratings, with the exception of the focal enlargement of the cortical sulci, exhibited moderate-to-substantial agreement.

High-convexity tightness (rated as $\geq 2$ ) and Sylvian fissure dilation (rated as $\geq 2$ ) were observed in $92 \%$ and $100 \%$ of patients, respectively. Focal enlargement of the cortical sulci and bumps in the lateral ventricular roof were present in $32 \%$ and $60 \%$ of the patients, respectively. Severe DWMHs and PVHs (rated as 3) were noted in $36 \%$ and $39 \%$ of the patients, respectively. The Evans indices were $>0.3$ in $82 \%$ of the patients. The callosal angles were $<90^{\circ}$ in $63 \%$ of patients.

\section{Presurgical Neuroimaging Findings that Predict the Shunt Response}

The results of the simple and multiple linear regression analyses are shown in Tables 4 and 5. Simple linear regression analysis demonstrated that presurgical high-convexity tightness was significantly associated with the 1-year changes in the iNPHGS total score (regression coefficient $[\mathrm{B}]=1.23$, coefficient of determination $\left.\left[R^{2}\right]=0.13, P=.004\right)$, the 1 -year changes in the iNPHGS gait score $\left(\mathrm{B}=0.59, R^{2}=0.16, P=.002\right)$, and the 1 -year changes in the MMSE $\left(\mathrm{B}=2.56, R^{2}=0.17, P=.001\right)$. There were significant associations between the presurgical callosal angle and the 1-year 
changes in the MMSE $\left(\mathrm{B}=-0.04, R^{2}=0.08, P=.035\right)$ and between presurgical Sylvian fissure dilation and the 1-year changes in the iNPHGS gait $\left(\mathrm{B}=0.59, R^{2}=0.08, P=.029\right)$.

A multiple linear regression analysis demonstrated that presurgical high-convexity tightness alone predicted the 1-year changes in the iNPHGS total score $\left(\mathrm{B}=0.99, R^{2}=0.24, P=.017\right)$ and the gait score $\left(\mathrm{B}=0.52, R^{2}=0.21, P=.006\right)$.

\section{DISCUSSION}

The primary focus of neuroimaging studies of hydrocephalus has been the differentiation of hydrocephalus from other neurologic diseases. Although ventriculomegaly is a primary morphologic feature of hydrocephalus, it is also observed in brain atrophy. Earlier studies claimed that the absence of Sylvian fissure dilation is a neuroimaging feature that differentiates hydrocephalus from brain atrophy. ${ }^{23,24}$ However, later studies suggested that Sylvian fissure dilation is present in most patients with iNPH. Thus, high-convexity tightness was subsequently proposed as an alternative feature for differentiating iNPH from brain atrophy. ${ }^{25}$ The combination of high-convexity tightness, Sylvian fissure dilation, and ventriculomegaly has been termed "disproportionately enlarged subarachnoid space hydrocephalus," and it has been increasingly recognized as a neuroimaging hallmark of iNPH. ${ }^{26}$ The value of DESH in differentiating iNPH from other neurologic diseases has been confirmed by several studies. ${ }^{27-30}$

Several diagnostic criteria for iNPH have recently been proposed. ${ }^{1,8}$ One recent study reported that the effectiveness of shunt surgery for patients who were diagnosed according to one of these guidelines remained at $62.7 \%$ in terms of the mRS. ${ }^{31}$ The incorporation of neuroimaging features that are predictive of surgical outcomes into the diagnostic criteria would thus improve the efficacy of shunt surgery. However, this issue has been systematically investigated in only a few studies. The current study demonstrates that high-convexity tightness, which is a component of $\mathrm{DESH}$, is the most predictive neuroimaging feature. Additionally, Sylvian fissure dilation and a small callosal angle were associated with better shunt responses.

The shunt response in patients with iNPH is governed by 2

Table 4: Results of simple linear regression analysis for presurgical neuroimaging features associated with surgical outcome: 1-year changes

\begin{tabular}{lccccccc}
\hline \multirow{1}{*}{\multicolumn{1}{c}{ Neuroimaging Findings }} & Total & Gait & Cognitive & Urinary & TUG & MMSE \\
\cline { 2 - 6 } & 7.34 & 1.00 & 0.97 & 5.37 & 60.96 & 9.22 \\
\hline Evans Index & -0.02 & -0.01 & -0.01 & -0.01 & -0.12 & $-0.04^{\mathrm{a}}$ \\
Callosal angle & $1.23^{\mathrm{b}}$ & $0.59^{\mathrm{b}}$ & 0.22 & 0.42 & -3.17 & $2.56^{\mathrm{b}}$ \\
High-convexity tightness & 1.03 & $0.59^{\mathrm{a}}$ & -0.09 & 0.53 & -2.65 & 1.00 \\
Sylvian fissure dilation & 0.71 & 0.19 & 0.00 & 0.53 & -2.25 & 1.19 \\
Focal enlargement of cortical sulci & 0.47 & 0.31 & 0.11 & 0.05 & 2.20 & 1.20 \\
Bumps in the lateral ventricle & -0.20 & -0.07 & 0.01 & -0.14 & 0.53 & 0.10 \\
DWMHs & -0.33 & -0.05 & -0.10 & -0.19 & 3.19 & -0.25 \\
PVHs & & & & & & \\
\hline
\end{tabular}

a $P<.05$.

${ }^{\mathrm{b}} \mathrm{P}<.01$. factors: namely, reversibility and comorbidity. Previous studies have demonstrated that symptomatic improvements following shunt surgery are associated with less severe symptoms and shorter disease duration, which suggest that reversibility declines as the disease progresses. The relationship between shunt response and brain tissue resilience is also supported by a previous neuroimaging study in which volume decreases of the lateral ventricle were found to be significantly correlated with symptomatic improvement. ${ }^{32}$ In agreement with these findings, recent studies have suggested that delayed shunt surgery is associated with poorer symptomatic improvement. ${ }^{31,33,34}$

In addition to reversibility, other neurologic comorbid diseases have a significant influence on shunt response. A recent positron-emission tomography study demonstrated that patients with significant cortical amyloid deposits exhibit less cognitive improvement following shunt surgery. ${ }^{35}$ High-convexity tightness or DESH is probably more strongly related to comorbidity than to reversibility. Elderly individuals who exhibit DESH on MR imaging can be asymptomatic for 5 or more years, ${ }^{36}$ which suggests that DESH is not well-correlated with pathophysiologic severity and reversibility. On the other hand, DESH or high-convexity tightness may be associated with the purity of iNPH pathology. Patients who exhibited weak typicality of DESH or mild highconvexity tightness may have comorbid pathologies with higher probabilities compared with those with typical DESH or severe high-convexity tightness. The results of our study may reflect this "typicality" effect.

Virhammar et $\mathrm{al}^{21}$ recently investigated the neuroimaging features predictive of shunt responses in 108 patients with iNPH. These authors demonstrated that DESH and a small callosal angle, not high-convexity tightness or Sylvian fissure dilation, were associated with better shunt responses. Although the study of Virhammar et al and our own agree about the importance of DESH, the studies also differ in some ways. Several factors may be associated with these discrepancies. First, the studies differed in their neuroimaging inclusion criteria. Virhammar et al used only ventriculomegaly for study inclusion, whereas the current study used high-convexity tightness, Sylvian fissure dilation, and ventriculomegaly. Because the pathophysiology of $\mathrm{iNPH}$ is presumably heterogeneous, these differences in the inclusion criteria may have led to a substantial bias. Second, the differences in the statistical procedures used in these studies should be noted. Univariate logistic regression with a dichotomous outcome measure (ie, the presence or absence of shunt response) was used in the study by Virhammar et al, whereas our study used multiple linear regression with ordinary outcome measures. Whether patients whose symptoms remain unchanged before

Table 5: Results of stepwise multiple linear regression analysis

\begin{tabular}{lllllccc}
\hline 1-Year Changes & Neuroimaging Findings & B & SE B & 95\% CI B & $\boldsymbol{\beta}$ & $\boldsymbol{R}^{2}$ & $\boldsymbol{P}$ Value \\
\hline iNPHGS total & High-convexity tightness & 0.99 & 0.40 & $0.18-1.80$ & 0.29 & 0.24 & .017 \\
iNPHGS gait & High-convexity tightness & 0.52 & 0.18 & $0.16-0.88$ & 0.35 & 0.21 & .006 \\
\hline
\end{tabular}

Note:-B indicates regression coefficient; $\beta$, standardized regression coefficient; $R^{2}$, coefficient of determination; SE B, standard error of the regression coefficient. 
and after surgery are assigned to the "responsive" or "unresponsive" group may have a significant impact on results. We argue that the lack of symptomatic deterioration for $\geq 1$-year observation probably should be interpreted as "responsive" because previous studies have demonstrated that conditions of patients with iNPH who did not receive surgical intervention deteriorated within a year. ${ }^{37,38}$

The current study has several limitations. First, patients with large cerebrovascular lesions and those strongly suspected of having neurodegenerative diseases were excluded from this study. Thus, the applicability of our findings to patients with other comorbid neurologic diseases is unknown. Second, the visual rating scale for the morphologic features of iNPH used in the current study has not been validated. Although we chose a visual inspection method because of its clinical utility, our rating system may be suboptimal. The validity of our findings should be examined in comparison with those based on other neuroimaging methods, such as MR imaging volumetry. Finally, this study was conducted in a single center and used a single MR imaging scanner. Future multicenter studies are needed to further verify the neuroimaging features that predict surgical outcome in iNPH.

\section{CONCLUSIONS}

We investigated the predictive values of neuroimaging features frequently observed in iNPH, including the Evans index, highconvexity tightness, Sylvian fissure dilation, callosal angle, focal enlargement of the cortical sulci, bumps in the lateral ventricular roof, and deep white matter and periventricular hyperintensities, for response to shunt surgery. Among them, high-convexity tightness was the best predictor of shunt response in iNPH.

Disclosures: Yoshiyuki Nishio-UNRELATED: Grants/Grants Pending: Ministry of Education, Culture, Sports, Science and Technology Japan, ${ }^{*}$ Comments: research grant for a study on cognitive problems in patients with epilepsy; Payment for Development of Educational Presentations: Eisai, Comments: educational lectures on dementia with Lewy bodies and other dementias. Toru Baba-UNRELATED: Payment for Lectures (including service on Speakers Bureaus): Boehringer Ingelheim, Novartis Pharmaceuticals, Eisai, Daiichi Sankyo, Kyowa Hakko Kirin, Dainippon Sumitomo Pharma. Etsuro Mori-RELATED: Grant: Ministry of Health, Labour and Welfare, Japan, Comments: Health and Labor Sciences Research Grants for Research on Intractable Diseases; UNRELATED: Payment for Lectures (including service on Speakers Bureaus): Johnson \& Johnson, Medtronic, Toshiba, Nihon Medi-Physics, Comments: Honoraria. *Money paid to the institution.

\section{REFERENCES}

1. Mori E, Ishikawa M, Kato T, et al. Guidelines for management of idiopathic normal pressure hydrocephalus: second edition. Neurol Med Chir (Tokyo) 2012;52:775-809 CrossRef Medline

2. Jaraj D, Rabiei K, Marlow T, et al. Prevalence of idiopathic normalpressure hydrocephalus. Neurology 2014;82:1449-54 CrossRef Medline

3. de Lau LM, Breteler MM. Epidemiology of Parkinson's disease. Lancet Neurol 2006;5:525-35 CrossRef Medline

4. Malm J, Graff-Radford NR, Ishikawa M, et al. Influence of comorbidities in idiopathic normal pressure hydrocephalus: research and clinical care - a report of the ISHCSF task force on comorbidities in INPH. Fluids Barriers CNS 2013;10:22 CrossRef Medline

5. Williams MA, Relkin NR. Diagnosis and management of idiopathic normal-pressure hydrocephalus. Neurol Clin Pract 2013;3:375-85 CrossRef Medline

6. Hebb AO, Cusimano MD. Idiopathic normal pressure hydrocephalus: a systematic review of diagnosis and outcome. Neurosurgery 2001;49:1166-84; discussion 1184-86 CrossRef Medline
7. Klinge P, Hellström P, Tans J, et al; European iNPH Multicentre Study Group. One-year outcome in the European multicentre study on iNPH. Acta Neurol Scand 2012;126:145-53 CrossRef Medline

8. Relkin N, Marmarou A, Klinge P, et al. Diagnosing idiopathic normal-pressure hydrocephalus. Neurosurgery 2005;57:S4-16; discussion ii-v Medline

9. Kahlon B, Sundbärg G, Rehncrona S. Comparison between the lumbar infusion and CSF tap tests to predict outcome after shunt surgery in suspected normal pressure hydrocephalus. J Neurol Neurosurg Psychiatry 2002;73:721-26 CrossRef Medline

10. Sand T, Bovim G, Grimse R, et al. Idiopathic normal pressure hydrocephalus: the CSF tap-test may predict the clinical response to shunting. Acta Neurol Scand 1994;89:311-16 Medline

11. Walchenbach R, Geiger E, Thomeer RT, et al. The value of temporary external lumbar CSF drainage in predicting the outcome of shunting on normal pressure hydrocephalus. J Neurol Neurosurg Psychiatry 2002;72:503-06 Medline

12. Ishikawa M, Hashimoto M, Mori E, et al. The value of the cerebrospinal fluid tap test for predicting shunt effectiveness in idiopathic normal pressure hydrocephalus. Fluids Barriers CNS 2012;9:1 CrossRef Medline

13. Eide PK, Brean A. Cerebrospinal fluid pulse pressure amplitude during lumbar infusion in idiopathic normal pressure hydrocephalus can predict response to shunting. Cerebrospinal Fluid Res 2010; 7:5 CrossRef Medline

14. Ishikawa M, Hashimoto M, Kuwana N, et al. Guidelines for management of idiopathic normal pressure hydrocephalus. Neurol Med Chir (Tokyo) 2008;48(suppl):S1-23 CrossRef Medline

15. Iseki C, Kawanami T, Nagasawa H, et al. Asymptomatic ventriculomegaly with features of idiopathic normal pressure hydrocephalus on MRI (AVIM) in the elderly: a prospective study in a Japanese population. J Neurol Sci 2009;277:54-57 CrossRef Medline

16. Kubo Y, Kazui H, Yoshida T, et al. Validation of grading scale for evaluating symptoms of idiopathic normal-pressure hydrocephalus. Dement Geriatr Cogn Disord 2008;25:37-45 CrossRef Medline

17. Podsiadlo D, Richardson S. The timed "Up \& Go": a test of basic functional mobility for frail elderly persons. J Am Geriatr Soc 1991; 39:142-48 CrossRef Medline

18. Mori E, Mitani Y, Yamadori A. Usefulness of Japanese version of the Mini-Mental State Examination in neurological patients [in Japanese]. Jpn J Neuropsychol 1985;1:82-90

19. Evans WA. An encephalographic ratio for estimating ventricular enlargement and cerebral atrophy. Arch NeurPsych 1942;47:931-37 CrossRef

20. Ishii K, Kanda T, Harada A, et al. Clinical impact of the callosal angle in the diagnosis of idiopathic normal pressure hydrocephalus. Eur Radiol 2008;18:2678-83 CrossRef Medline

21. Virhammar J, Laurell K, Cesarini KG, et al. Preoperative prognostic value of MRI findings in 108 patients with idiopathic normal pressure hydrocephalus. AJNR Am J Neuroradiol 2014;35:2311-18 CrossRef Medline

22. Fazekas F, Chawluk JB, Alavi A, et al. MR signal abnormalities at $\mathbf{1 . 5}$ T in Alzheimer's dementia and normal aging. AJR Am J Roentgenol 1987;149:351-56 CrossRef Medline

23. Gunasekera L, Richardson AE. Computerized axial tomography in idiopathic hydrocephalus. Brain 1977;100:749-54 CrossRef Medline

24. Benzel EC, Pelletier AL, Levy PG. Communicating hydrocephalus in adults: prediction of outcome after ventricular shunting procedures. Neurosurgery 1990;26:655-60 CrossRef Medline

25. Kitagaki H, Mori E, Ishii K, et al. CSF spaces in idiopathic normal pressure hydrocephalus: morphology and volumetry. AJNR Am J Neuroradiol 1998;19:1277-84 Medline

26. Hashimoto M, Ishikawa M, Mori E, et al; Study of INPH on neurological improvement (SINPHONI). Diagnosis of idiopathic normal pressure hydrocephalus is supported by MRI-based scheme: a prospective cohort study. Cerebrospinal Fluid Res 2010;7:18 CrossRef Medline

27. Yamashita F, Sasaki M, Takahashi S, et al. Detection of changes in 
cerebrospinal fluid space in idiopathic normal pressure hydrocephalus using voxel-based morphometry. Neuroradiology 2010;52: 381-86 CrossRef Medline

28. Yamashita F, Sasaki M, Saito M, et al. Voxel-based morphometry of disproportionate cerebrospinal fluid space distribution for the differential diagnosis of idiopathic normal pressure hydrocephalus. J Neuroimaging 2014;24:359-65 CrossRef Medline

29. Ishii K, Soma T, Shimada K, et al. Automatic volumetry of the cerebrospinal fluid space in idiopathic normal pressure hydrocephalus. Dement Geriatr Cogn Dis Extra 2013;3:489-96 CrossRef Medline

30. Kojoukhova M, Koivisto AM, Korhonen R, et al. Feasibility of radiological markers in idiopathic normal pressure hydrocephalus. Acta Neurochir (Wien) 2015;157:1709-18; discussion 1719 CrossRef Medline

31. Kazui H, Miyajima M, Mori E, et al; SINPHONI-2 Investigators. Lumboperitoneal shunt surgery for idiopathic normal pressure hydrocephalus (SINPHONI-2): an open-label randomised trial. Lancet Neurol 2015;14:585-94 CrossRef Medline

32. Hiraoka K, Yamasaki H, Takagi M, et al. Changes in the volumes of the brain and cerebrospinal fluid spaces after shunt surgery in idiopathic normal-pressure hydrocephalus. J Neurol Sci 2010;296: 7-12 CrossRef Medline
33. Caruso R, Cervoni L, Vitale AM, et al. Idiopathic normal-pressure hydrocephalus in adults: result of shunting correlated with clinical findings in 18 patients and review of the literature. Neurosurg Rev 1997;20:104-07 CrossRef Medline

34. Andrén K, Wikkelso C, Tisell M, et al. Natural course of idiopathic normal pressure hydrocephalus. J Neurol Neurosurg Psychiatry 2014; 85:806-10 CrossRef Medline

35. Hiraoka K, Narita W, Kikuchi H, et al. Amyloid deposits and response to shunt surgery in idiopathic normal-pressure hydrocephalus. J Neurol Sci 2015;356:124-28 CrossRef Medline

36. Iseki $\mathrm{C}$, Takahashi $Y$, Wada $\mathrm{M}$, et al. Incidence of idiopathic normal pressure hydrocephalus (iNPH): a 10-year follow-up study of a rural community in Japan. J Neurol Sci 2014;339:108-12 CrossRef Medline

37. Razay G, Vreugdenhil A, Liddell J. A prospective study of ventriculoperitoneal shunting for idiopathic normal pressure hydrocephalus. J Clin Neurosci 2009;16:1180-83 CrossRef Medline

38. Scollato A, Tenenbaum R, Bahl G, et al. Changes in aqueductal CSF stroke volume and progression of symptoms in patients with unshunted idiopathic normal pressure hydrocephalus. AJNR Am J Neuroradiol 2008;29:192-97 CrossRef Medline 